\title{
Azide reactions for controlling clean silicon surface chemistry: Benzylazide
}

on Si(100)-2x1.

Semyon Bocharov, Olga Dmytrenko, Lucila P. Méndez De Leo, and Andrew V. Teplyakov*

Department of Chemistry and Biochemistry, University of Delaware, Newark, DE 10716

Supporting Information. 
All the computational studies reported here were performed using the B3LYP hybrid density functional, ${ }^{1,2}$ with the $6-311+\mathrm{G}(\mathrm{d}, \mathrm{p})$ basis set, as implemented in the Gaussian 03 suite of programs. ${ }^{3}$ GridChem, Computational Chemistry Grid, ${ }^{4,5}$ (www.gridchem.org) is acknowledged for computational resources and services for transition state results used in this publication.

(1) Becke, A. D., J. Chem. Phys. 1993, 98, 1372-1377.

(2) Lee, C.; Yang, W.; Parr, R. G., Phys. Rev. B 1988, 37, 785.

(3) Frisch, M. J. T., Trucks, G. W.; Schlegel, H. B.; Scuseria, G. E.; Robb, M. A.; Cheeseman, J. R.; Montgomery, Jr., J. A.; Vreven, T.; Kudin, K. N.; Burant, J. C.; Millam, J. M.; Iyengar, S. S.; Tomasi, J.; Barone, V.; Mennucci, B.; Cossi, M.; Scalmani, G.; Rega, N.; Petersson, G. A.; Nakatsuji, H.; Hada, M.; Ehara, M.; Toyota, K.; Fukuda, R.; Hasegawa, J.; Ishida, M.; Nakajima, T.; Honda, Y.; Kitao, O.; Nakai, H.; Klene, M.; Li, X.; Knox, J. E.; Hratchian, H. P.; Cross, J. B.; Bakken, V.; Adamo, C.; Jaramillo, J.; Gomperts, R.; Stratmann, R. E.; Yazyev, O.; Austin, A. J.; Cammi, R.; Pomelli, C.; Ochterski, J. W.; Ayala, P. Y.; Morokuma, K.; Voth, G. A.; Salvador, P.; Dannenberg, J. J.; Zakrzewski, V. G.; Dapprich, S.; Daniels, A. D.; Strain, M. C.; Farkas, O.; Malick, D. K.; Rabuck, A. D.; Raghavachari, K.; Foresman, J. B.; Ortiz, J. V.; Cui, Q.; Baboul, A. G.; Clifford, S.; Cioslowski, J.; Stefanov, B. B.; Liu, G.; Liashenko, A.; Piskorz, P.; Komaromi, I.; Martin, R. L.; Fox, D. J.; Keith, T.; Al-Laham, M. A.; Peng, C. Y.; Nanayakkara, A.; Challacombe, M.; Gill, P. M. W.; Johnson, B.; Chen, W.; Wong, M. W.; Gonzalez, C.; and Pople, J. A. Gaussian 03, Revision C.02, Gaussian, Inc.: Wallingford CT, 2004.

(4) Milfeld, K.; Guiang, C.; Pamidighantam, S.; Giuliani, J. "Cluster Computing through an Application-oriented Computational Chemistry Grid." Proceedings of the 2005 Linux Clusters: The HPC Revolution.

(5) Dooley, R.; Milfeld, K.; Guiang, C.; Pamidighantam, S.; Allen, G. "From Proposal to Production: Lessons Learned Developing the Computational Chemistry Grid Cyberinfrastructure." Proceedings of the Global Grid Forum 14, 2005. 


\section{Nitrogen molecule, $\mathrm{N}_{2}$}

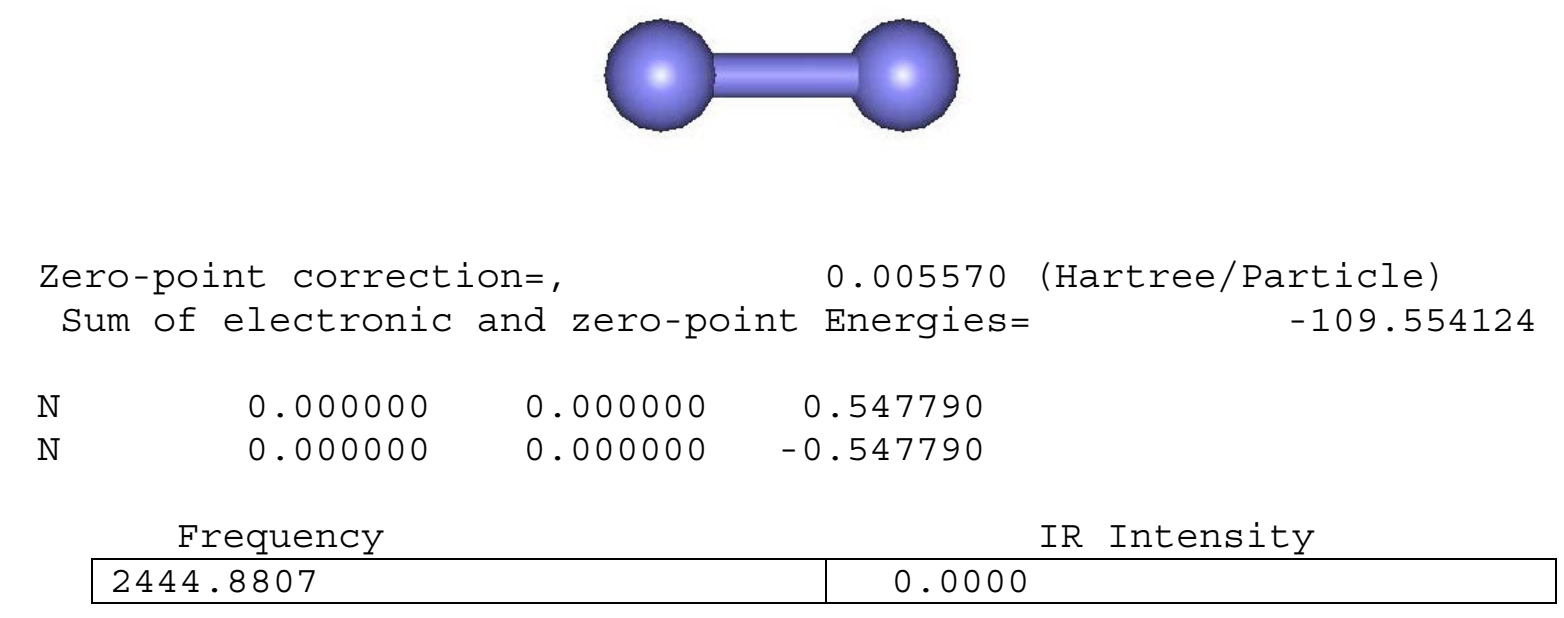




\section{Benzylazide}
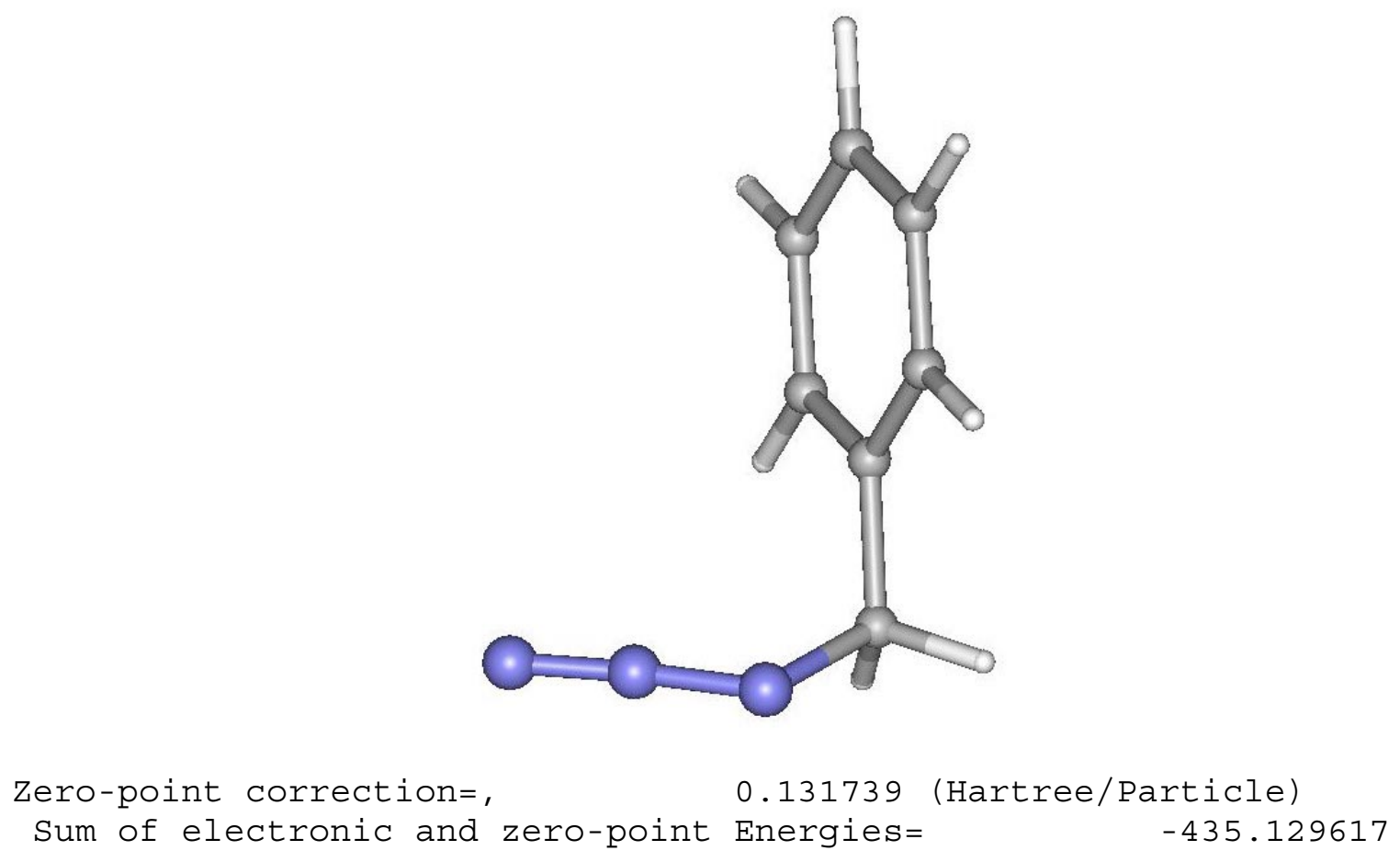

$\begin{array}{lrrr}\mathrm{C} & -0.208183 & -0.397426 & 0.524270 \\ \mathrm{C} & -0.717888 & 0.858768 & 0.862792 \\ \mathrm{C} & -1.959960 & 1.274726 & 0.383024 \\ \mathrm{C} & -2.703936 & 0.436075 & -0.442668 \\ \mathrm{C} & -2.202907 & -0.820305 & -0.786706 \\ \mathrm{C} & -0.964028 & -1.233264 & -0.306306 \\ \mathrm{H} & -0.144328 & 1.515203 & 1.509496 \\ \mathrm{H} & -2.343760 & 2.251453 & 0.655055 \\ \mathrm{H} & -3.669955 & 0.756759 & -0.815818 \\ \mathrm{H} & -2.779844 & -1.478149 & -1.426958 \\ \mathrm{H} & -0.575747 & -2.209314 & -0.578219 \\ \mathrm{~N} & 2.817403 & 1.283323 & -0.956858 \\ \mathrm{~N} & 2.481741 & 0.315429 & -0.468384 \\ \mathrm{~N} & 2.213067 & -0.798197 & -0.020247 \\ \mathrm{C} & 1.147982 & -0.839358 & 1.024070 \\ \mathrm{H} & 1.125996 & -1.881393 & 1.340673 \\ \mathrm{H} & 1.455678 & -0.233740 & 1.883345\end{array}$

Frequency

\begin{tabular}{|r|l|}
\hline 30.3466 & 0.2525 \\
\hline 46.1133 & 0.2082 \\
\hline 142.4332 & 3.5998 \\
\hline 197.1825 & 1.7453 \\
\hline 324.9415 & 3.0405 \\
\hline 342.7580 & 1.4948 \\
\hline
\end{tabular}




\begin{tabular}{|c|c|}
\hline 412.8750 & 0.1179 \\
\hline 490.5747 & 4.9182 \\
\hline 563.1134 & 5.8903 \\
\hline 578.4710 & 13.9771 \\
\hline 635.2577 & 0.0130 \\
\hline 681.7203 & 28.0926 \\
\hline 712.3217 & 54.2890 \\
\hline 764.5530 & 26.7432 \\
\hline 825.5461 & 3.7188 \\
\hline 858.4041 & 0.0755 \\
\hline 888.1687 & 25.5307 \\
\hline 937.1489 & 0.8467 \\
\hline 980.2822 & 6.8080 \\
\hline 987.8638 & 0.0246 \\
\hline 1008.5694 & 0.0029 \\
\hline 1017.8212 & 0.4537 \\
\hline 1049.5449 & 2.7548 \\
\hline 1108.2438 & 3.6961 \\
\hline 1183.2469 & 0.0359 \\
\hline 1203.3242 & 0.6122 \\
\hline 1220.1131 & 7.7189 \\
\hline 1233.7077 & 0.2494 \\
\hline 1299.6977 & 151.3378 \\
\hline 1342.9464 & 8.5103 \\
\hline 1361.7215 & 3.5021 \\
\hline 1382.3854 & 14.9570 \\
\hline 1484.0746 & 5.9339 \\
\hline 1497.4471 & 6.0584 \\
\hline 1526.4231 & 4.7748 \\
\hline 1625.2196 & 0.8931 \\
\hline 1643.4184 & 0.5586 \\
\hline 2227.6659 & 520.3926 \\
\hline 3030.1462 & 36.6572 \\
\hline 3115.2693 & 8.2303 \\
\hline 3158.4980 & 5.6034 \\
\hline 3162.4243 & 1.1998 \\
\hline 3172.0162 & 6.5560 \\
\hline 3181.3146 & 24.1164 \\
\hline 3191.5179 & 11.6192 \\
\hline
\end{tabular}




\section{Benzylazide, dimer}

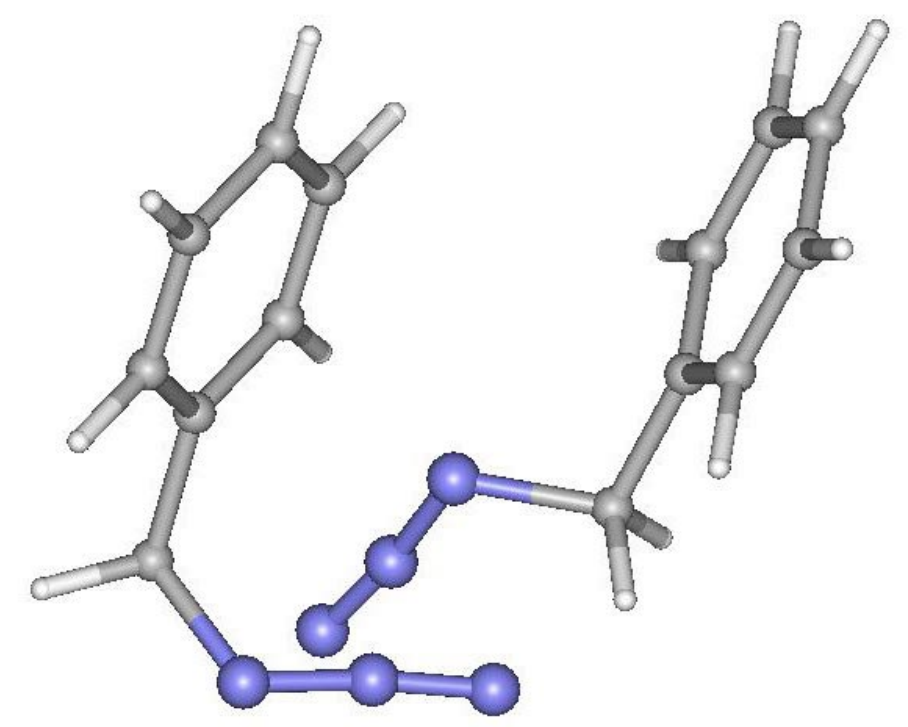

Zero-point correction=, 0.263740 (Hartree/Particle) Sum of electronic and zero-point Energies= $-870.261052$

The dimer is more stable than two single molecules of benzylazide by $5.46 \mathrm{~kJ} / \mathrm{mol}$ (or by $4.77 \mathrm{~kJ} / \mathrm{mol}$ is the zero-point correction is taken into account).

$\begin{array}{lrrr}\mathrm{C} & -2.652293 & 0.041575 & 0.329384 \\ \mathrm{C} & -3.494005 & -0.496897 & -0.649865 \\ \mathrm{C} & -3.830604 & -1.847088 & -0.628368 \\ \mathrm{C} & -3.329506 & -2.676464 & 0.376300 \\ \mathrm{C} & -2.490828 & -2.148204 & 1.354411 \\ \mathrm{C} & -2.152392 & -0.795011 & 1.329171 \\ \mathrm{H} & -3.885113 & 0.144323 & -1.433179 \\ \mathrm{H} & -4.485865 & -2.252504 & -1.391248 \\ \mathrm{H} & -3.594447 & -3.727682 & 0.395676 \\ \mathrm{H} & -2.096331 & -2.786914 & 2.136574 \\ \mathrm{H} & -1.492140 & -0.388153 & 2.087520 \\ \mathrm{~N} & -1.170307 & 4.018623 & -1.588986 \\ \mathrm{~N} & -1.305177 & 2.968113 & -1.178665 \\ \mathrm{~N} & -1.369509 & 1.790957 & -0.830094 \\ \mathrm{C} & -2.295967 & 1.506585 & 0.309538 \\ \mathrm{H} & -3.198659 & 2.117885 & 0.199824 \\ \mathrm{H} & -1.800577 & 1.787977 & 1.243070 \\ \mathrm{C} & 2.783988 & -0.110803 & -0.460766 \\ \mathrm{C} & 1.550179 & -0.625174 & -0.870902 \\ \mathrm{C} & 1.384742 & -1.997800 & -1.056617 \\ \mathrm{C} & 2.451200 & -2.866313 & -0.836808 \\ \mathrm{C} & 3.685895 & -2.360169 & -0.428163 \\ \mathrm{C} & 3.849906 & -0.990836 & -0.240616 \\ \mathrm{H} & 0.715417 & 0.045982 & -1.047293 \\ \mathrm{H} & 0.422146 & -2.383690 & -1.372065\end{array}$




$\begin{array}{lrrr}\mathrm{H} & 2.323766 & -3.932924 & -0.984471 \\ \mathrm{H} & 4.519880 & -3.032164 & -0.259028 \\ \mathrm{H} & 4.810204 & -0.599693 & 0.079732 \\ \mathrm{~N} & 0.807326 & 1.481474 & 2.344550 \\ \mathrm{~N} & 1.765265 & 1.593696 & 1.743285 \\ \mathrm{~N} & 2.855588 & 1.766565 & 1.209743 \\ \mathrm{C} & 2.956919 & 1.371657 & -0.229756 \\ \mathrm{H} & 3.951930 & 1.700691 & -0.525307 \\ \mathrm{H} & 2.224093 & 1.940527 & -0.810280\end{array}$

Frequency

\begin{tabular}{|c|c|}
\hline & \\
\hline 11.1284 & 0.3796 \\
\hline 12.9577 & 0.1694 \\
\hline 16.7720 & 0.0959 \\
\hline 21.6415 & 0.5090 \\
\hline 29.3252 & 0.0958 \\
\hline 31.9281 & 0.6200 \\
\hline 38.0494 & 0.7217 \\
\hline 41.8077 & 0.3139 \\
\hline 53.9960 & 1.1936 \\
\hline 65.2015 & 3.1735 \\
\hline 109.7994 & 3.1177 \\
\hline 139.8126 & 2.9812 \\
\hline 200.3758 & 1.9129 \\
\hline 205.0369 & 2.7440 \\
\hline 286.0253 & 2.2821 \\
\hline 326.9647 & 2.4295 \\
\hline 335.9658 & 3.3502 \\
\hline 338.0011 & 1.2203 \\
\hline 413.3611 & 0.1594 \\
\hline 415.5351 & 0.1349 \\
\hline 481.7085 & 4.5302 \\
\hline 492.0905 & 4.0262 \\
\hline 554.3273 & 6.7267 \\
\hline 564.6538 & 3.8305 \\
\hline 578.0129 & 11.1834 \\
\hline 589.9357 & 8.0325 \\
\hline 634.3406 & 1.8886 \\
\hline 634.7479 & 0.2179 \\
\hline 652.9521 & 22.5044 \\
\hline 679.5903 & 29.5687 \\
\hline 711.5124 & 54.7878 \\
\hline 714.5697 & 41.5881 \\
\hline 764.7403 & 48.4134 \\
\hline 766.2924 & 23.1337 \\
\hline 826.3537 & 4.0645 \\
\hline 842.9704 & 16.7321 \\
\hline 859.4392 & 0.5787 \\
\hline 864.1594 & 0.4636 \\
\hline 881.1088 & 25.7567 \\
\hline
\end{tabular}




\begin{tabular}{|c|c|}
\hline 908.2340 & 17.6356 \\
\hline 933.9104 & 2.1714 \\
\hline 944.6670 & 1.1793 \\
\hline 975.0974 & 6.5439 \\
\hline 976.4595 & 2.0859 \\
\hline 990.4721 & 0.1789 \\
\hline 993.4724 & 0.0087 \\
\hline 1009.4410 & 0.0271 \\
\hline 1009.6968 & 0.0969 \\
\hline 1017.1596 & 0.1934 \\
\hline 1018.2473 & 0.5754 \\
\hline 1049.4482 & 3.6951 \\
\hline 1049.9542 & 2.1776 \\
\hline 1107.1132 & 4.6613 \\
\hline 1110.2121 & 2.7040 \\
\hline 1182.7262 & 0.0954 \\
\hline 1183.0219 & 0.0655 \\
\hline 1204.4602 & 0.9046 \\
\hline 1206.4362 & 0.3489 \\
\hline 1222.7484 & 6.4253 \\
\hline 1229.7320 & 0.8219 \\
\hline 1232.7551 & 4.1889 \\
\hline 1234.4449 & 0.6155 \\
\hline 1304.9647 & 130.9131 \\
\hline 1318.8214 & 208.8523 \\
\hline 1340.0017 & 16.6339 \\
\hline 1342.5124 & 8.7914 \\
\hline 1358.4043 & 11.7593 \\
\hline 1365.3470 & 0.8422 \\
\hline 1380.5496 & 40.7950 \\
\hline 1385.8349 & 22.0195 \\
\hline 1484.2683 & 6.5024 \\
\hline 1484.6390 & 7.1651 \\
\hline 1496.0067 & 9.3598 \\
\hline 1497.0536 & 5.0395 \\
\hline 1527.6142 & 3.9778 \\
\hline 1529.1800 & 4.9381 \\
\hline 1624.6775 & 0.4873 \\
\hline 1626.8831 & 0.3622 \\
\hline 1642.9875 & 0.1137 \\
\hline 1646.3705 & 0.4272 \\
\hline 2222.2018 & 528.0733 \\
\hline 2229.5060 & 761.4856 \\
\hline 3017.8695 & 25.4733 \\
\hline 3041.6136 & 27.4202 \\
\hline 3070.6800 & 10.0317 \\
\hline 3121.8193 & 9.6348 \\
\hline 3158.4846 & 2.4973 \\
\hline 3159.3137 & 2.5751 \\
\hline
\end{tabular}




\begin{tabular}{|r|r|}
\hline 3161.3059 & 1.1561 \\
\hline 3165.6304 & 0.9909 \\
\hline 3169.7001 & 6.9817 \\
\hline 3173.0861 & 8.7806 \\
\hline 3181.1168 & 21.8810 \\
\hline 3181.6370 & 18.7802 \\
\hline 3190.5603 & 7.1509 \\
\hline 3190.7302 & 17.7043 \\
\hline
\end{tabular}




\section{Silicon Cluster}

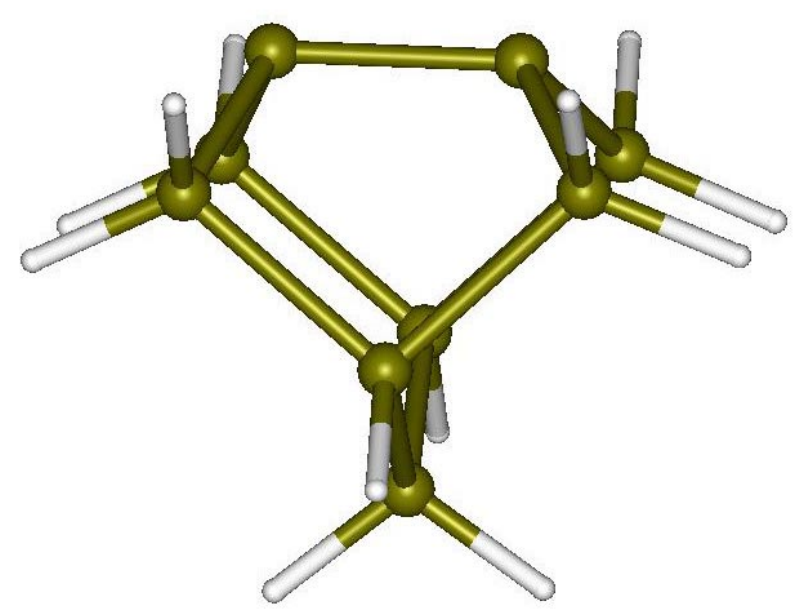

Zero-point correction=,

0.109511 (Hartree/Particle)

Sum of electronic and zero-point Energies=

$-2612.773716$

$\begin{array}{lrrr}\text { Si } & 2.038960 & -0.094369 & 1.095599 \\ \mathrm{Si} & -0.000021 & -0.198458 & 2.319408 \\ \mathrm{Si} & 1.980135 & 1.805548 & -0.328865 \\ \mathrm{Si} & 1.990316 & -1.748412 & -0.611818 \\ \mathrm{Si} & -2.038984 & -0.094370 & 1.095569 \\ \mathrm{Si} & 0.000010 & -0.951001 & -1.554804 \\ \mathrm{Si} & 0.000007 & 1.266130 & -1.473281 \\ \mathrm{Si} & -1.990297 & -1.748425 & -0.611830 \\ \mathrm{Si} & -1.980128 & 1.805555 & -0.328882 \\ \mathrm{H} & -1.867366 & -3.153208 & -0.136621 \\ \mathrm{H} & -3.172899 & -1.648668 & -1.508971 \\ \mathrm{H} & 3.177398 & 1.850692 & -1.212153 \\ \mathrm{H} & 1.872471 & 3.112190 & 0.376934 \\ \mathrm{H} & -3.177386 & 1.850736 & -1.212173 \\ \mathrm{H} & -1.872437 & 3.112187 & 0.376934 \\ \mathrm{H} & -0.000028 & 0.918571 & 3.309108 \\ \mathrm{H} & -0.000024 & -1.467346 & 3.105114 \\ \mathrm{H} & -3.179614 & -0.182047 & 2.066003 \\ \mathrm{H} & 3.179572 & -0.182046 & 2.066054 \\ \mathrm{H} & 1.867409 & -3.153206 & -0.136635 \\ \mathrm{H} & 3.172925 & -1.648615 & -1.508945\end{array}$

Frequency

\begin{tabular}{|r|r|}
\hline 36.2851 & 18.6657 \\
\hline 83.7677 & 0.4649 \\
\hline 84.6724 & 0.0037 \\
\hline 93.7095 & 0.3579 \\
\hline 117.9608 & 8.6009 \\
\hline 124.4923 & 2.1075 \\
\hline
\end{tabular}




\begin{tabular}{|c|c|}
\hline 133.6306 & 1.9877 \\
\hline 161.7458 & 0.0180 \\
\hline 193.5979 & 1.3550 \\
\hline 252.2268 & 0.0239 \\
\hline 318.8669 & 0.9577 \\
\hline 331.5235 & 0.5035 \\
\hline 355.5585 & 0.4233 \\
\hline 362.3905 & 3.7386 \\
\hline 386.2071 & 2.3297 \\
\hline 394.2494 & 0.0004 \\
\hline 405.7457 & 0.0540 \\
\hline 410.2303 & 0.2553 \\
\hline 411.8656 & 0.0223 \\
\hline 417.3724 & 0.0206 \\
\hline 458.7407 & 2.3642 \\
\hline 459.1331 & 0.4519 \\
\hline 466.2020 & 2.5272 \\
\hline 477.4221 & 2.7572 \\
\hline 486.3518 & 0.2633 \\
\hline 546.6419 & 0.7378 \\
\hline 584.7595 & 0.0535 \\
\hline 595.5554 & 1.6823 \\
\hline 602.0454 & 0.2239 \\
\hline 617.5646 & 0.0916 \\
\hline 632.9784 & 5.6907 \\
\hline 638.1597 & 0.0047 \\
\hline 642.5869 & 1.0709 \\
\hline 650.9806 & 0.4190 \\
\hline 668.3262 & 12.1065 \\
\hline 670.1653 & 10.7849 \\
\hline 698.4051 & 0.0419 \\
\hline 705.3900 & 0.2017 \\
\hline 735.3947 & 173.2769 \\
\hline 744.6232 & 261.2583 \\
\hline 926.7438 & 129.8974 \\
\hline 928.1330 & 4.6352 \\
\hline 930.5271 & 321.2262 \\
\hline 932.1946 & 194.5274 \\
\hline 941.3069 & 0.6015 \\
\hline 2133.9489 & 158.8670 \\
\hline 2134.6242 & 240.0746 \\
\hline 2177.5804 & 157.1218 \\
\hline 2187.4192 & 22.1161 \\
\hline 2191.9589 & 85.5463 \\
\hline 2193.7300 & 225.8033 \\
\hline 2197.3533 & 145.6993 \\
\hline 2200.6173 & 31.5301 \\
\hline 2204.6537 & 37.5738 \\
\hline 2206.9902 & 143.7341 \\
\hline
\end{tabular}


2211.2590

2213.2054
56.1619

107.9748 


\section{Int1}

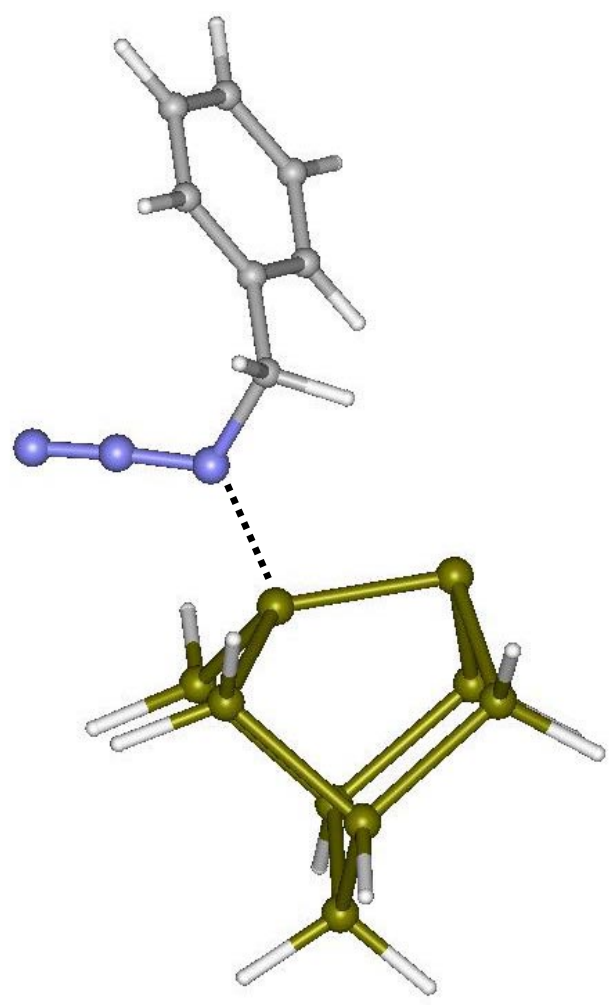

Zero-point correction=, 0.242248 (Hartree/Particle)

Sum of electronic and zero-point Energies= $-3047.923399$

$\begin{array}{lrrr}\text { Si } & -2.787563 & 1.673254 & 1.414582 \\ \mathrm{Si} & -4.317999 & -0.012159 & 0.746060 \\ \mathrm{Si} & -1.760628 & 2.626704 & -0.494693 \\ \mathrm{Si} & -0.870721 & 0.612893 & 2.302755 \\ \mathrm{Si} & -3.390954 & -1.521823 & -0.830677 \\ \mathrm{Si} & -0.290990 & -0.413592 & 0.253503 \\ \mathrm{Si} & -0.491593 & 0.959116 & -1.652808 \\ \mathrm{Si} & -1.452219 & -2.471805 & 0.128691 \\ \mathrm{Si} & -2.310517 & -0.309481 & -2.555096 \\ \mathrm{H} & -1.607316 & -3.143606 & 1.449940 \\ \mathrm{H} & -0.783871 & -3.431288 & -0.798745 \\ \mathrm{H} & -0.888436 & 3.741394 & -0.013192 \\ \mathrm{H} & -2.779409 & 3.246589 & -1.392825 \\ \mathrm{H} & -1.831544 & -1.309563 & -3.557835 \\ \mathrm{H} & -3.277158 & 0.575108 & -3.270612 \\ \mathrm{H} & -5.511516 & 0.644080 & 0.138527 \\ \mathrm{H} & -4.794796 & -0.761875 & 1.945137 \\ \mathrm{H} & -4.407723 & -2.554314 & -1.201988 \\ \mathrm{H} & -3.438643 & 2.579139 & 2.411304 \\ \mathrm{H} & -1.091331 & -0.358784 & 3.410455 \\ \mathrm{H} & 0.158646 & 1.596792 & 2.747714\end{array}$




$\begin{array}{lrrr}\mathrm{H} & 7.305719 & 1.545515 & 0.023575 \\ \mathrm{C} & 6.359006 & 1.044834 & -0.143949 \\ \mathrm{C} & 5.201199 & 1.794684 & -0.357490 \\ \mathrm{C} & 6.298643 & -0.346463 & -0.153249 \\ \mathrm{C} & 3.985331 & 1.155724 & -0.577215 \\ \mathrm{H} & 5.248151 & 2.877520 & -0.359863 \\ \mathrm{C} & 5.080122 & -0.988260 & -0.370876 \\ \mathrm{H} & 7.196635 & -0.932307 & 0.005034 \\ \mathrm{C} & 3.916016 & -0.243413 & -0.583001 \\ \mathrm{H} & 3.087066 & 1.737273 & -0.755971 \\ \mathrm{H} & 5.038191 & -2.072859 & -0.388685 \\ \mathrm{C} & 2.601553 & -0.931763 & -0.825129 \\ \mathrm{~N} & 1.670866 & -0.878967 & 0.372453 \\ \mathrm{H} & 2.731726 & -1.981874 & -1.095968 \\ \mathrm{H} & 2.013852 & -0.428804 & -1.597365 \\ \mathrm{~N} & 2.153174 & -1.283528 & 1.451766 \\ \mathrm{~N} & 2.519549 & -1.620889 & 2.460692\end{array}$

Frequency

\begin{tabular}{|c|c|}
\hline & \\
\hline 13.8938 & 0.2448 \\
\hline 15.1247 & 0.1637 \\
\hline 32.9233 & 0.5209 \\
\hline 39.8843 & 0.2585 \\
\hline 63.7332 & 0.4503 \\
\hline 68.7465 & 0.0036 \\
\hline 86.7004 & 0.3369 \\
\hline 93.8063 & 1.1076 \\
\hline 98.3607 & 0.6992 \\
\hline 103.9518 & 1.3534 \\
\hline 119.9420 & 0.7862 \\
\hline 130.9856 & 4.5017 \\
\hline 143.0322 & 0.8730 \\
\hline 146.1849 & 2.9471 \\
\hline 164.0913 & 1.5847 \\
\hline 188.6855 & 0.9095 \\
\hline 200.6006 & 1.0405 \\
\hline 220.6728 & 1.0102 \\
\hline 247.0173 & 0.6330 \\
\hline 274.7512 & 7.2993 \\
\hline 317.2312 & 17.3162 \\
\hline 325.4048 & 3.7493 \\
\hline 343.9952 & 33.5064 \\
\hline 351.7782 & 1.0092 \\
\hline 361.0242 & 2.0057 \\
\hline 367.2691 & 77.0246 \\
\hline 380.5858 & 0.0775 \\
\hline 393.5543 & 0.3676 \\
\hline 397.2097 & 0.0712 \\
\hline 412.6007 & 0.4635 \\
\hline
\end{tabular}




\begin{tabular}{|c|c|}
\hline 413.4628 & 0.4852 \\
\hline 419.2206 & 2.5706 \\
\hline 420.9813 & 0.5084 \\
\hline 427.7437 & 3.4505 \\
\hline 436.3302 & 0.1637 \\
\hline 451.8316 & 1.0071 \\
\hline 478.1884 & 0.3359 \\
\hline 479.8384 & 1.5590 \\
\hline 490.2552 & 6.6341 \\
\hline 509.1640 & 0.6583 \\
\hline 538.1875 & 4.8855 \\
\hline 559.5333 & 22.3596 \\
\hline 566.1711 & 3.9187 \\
\hline 591.8646 & 0.1829 \\
\hline 595.0178 & 1.1064 \\
\hline 600.0739 & 1.9981 \\
\hline 628.2937 & 9.4096 \\
\hline 629.4667 & 6.8934 \\
\hline 634.1691 & 0.4376 \\
\hline 637.1371 & 0.4182 \\
\hline 648.7207 & 23.6046 \\
\hline 668.6239 & 5.3543 \\
\hline 675.4243 & 6.8187 \\
\hline 676.7814 & 47.9377 \\
\hline 681.7237 & 8.2620 \\
\hline 701.5293 & 6.7008 \\
\hline 713.7194 & 47.8969 \\
\hline 750.3401 & 254.6274 \\
\hline 753.1095 & 268.0940 \\
\hline 771.7155 & 39.4675 \\
\hline 826.2652 & 7.9809 \\
\hline 863.2962 & 2.8528 \\
\hline 872.4182 & 98.0072 \\
\hline 927.3437 & 11.3532 \\
\hline 930.5369 & 134.0513 \\
\hline 932.2777 & 241.2466 \\
\hline 935.6421 & 169.8263 \\
\hline 942.7356 & 4.2283 \\
\hline 946.0347 & 26.7981 \\
\hline 985.3087 & 9.9458 \\
\hline 994.4609 & 0.0046 \\
\hline 1015.7015 & 0.0730 \\
\hline 1017.4972 & 0.2629 \\
\hline 1049.1484 & 2.9160 \\
\hline 1110.2937 & 4.1288 \\
\hline 1185.9837 & 0.3037 \\
\hline 1203.9335 & 9.7947 \\
\hline 1212.4049 & 60.1719 \\
\hline 1226.5132 & 11.2047 \\
\hline
\end{tabular}




\begin{tabular}{|c|c|}
\hline 1258.6353 & 32.7560 \\
\hline 1344.6802 & 2.7935 \\
\hline 1363.5711 & 1.0335 \\
\hline 1391.8952 & 11.8284 \\
\hline 1485.7212 & 7.9273 \\
\hline 1487.5977 & 10.2341 \\
\hline 1526.5169 & 9.5115 \\
\hline 1624.3167 & 1.5498 \\
\hline 1643.0445 & 0.8076 \\
\hline 2152.8027 & 128.0018 \\
\hline 2155.5526 & 36.1686 \\
\hline 2160.6734 & 265.5299 \\
\hline 2162.1328 & 128.5819 \\
\hline 2175.6232 & 3.1031 \\
\hline 2177.3652 & 137.2780 \\
\hline 2179.2641 & 179.7378 \\
\hline 2181.6463 & 172.4868 \\
\hline 2183.4441 & 54.3411 \\
\hline 2190.3918 & 139.9755 \\
\hline 2191.3249 & 33.5552 \\
\hline 2196.6746 & 144.0331 \\
\hline 2255.4631 & 547.6449 \\
\hline 3036.2749 & 68.7917 \\
\hline 3096.2437 & 20.6911 \\
\hline 3159.0435 & 4.8707 \\
\hline 3167.7069 & 0.0948 \\
\hline 3175.5460 & 2.9685 \\
\hline 3186.1428 & 12.1067 \\
\hline 3195.2250 & 13.9669 \\
\hline
\end{tabular}

Deuterium-substituted:

Frequency

\begin{tabular}{|r|r|}
\multicolumn{1}{|c|}{ Frequency } & IR Intensity \\
\hline 13.6025 & 0.1812 \\
\hline 14.7750 & 0.2040 \\
\hline 32.5783 & 0.5131 \\
\hline 38.8699 & 0.2243 \\
\hline 60.2318 & 0.4289 \\
\hline 66.3756 & 0.0059 \\
\hline 80.6564 & 0.2614 \\
\hline 87.9941 & 1.3285 \\
\hline 92.0142 & 0.2325 \\
\hline 107.7816 & 2.0892 \\
\hline 115.3122 & 0.8365 \\
\hline 138.5456 & 2.5564 \\
\hline 139.9378 & 0.6271 \\
\hline 162.0414 & 1.8808 \\
\hline 183.5565 & 1.3784 \\
\hline 198.6054 & 0.6020 \\
\hline 218.9159 & 1.1612 \\
\hline & 0.8887 \\
\hline
\end{tabular}




\begin{tabular}{|c|c|}
\hline 243.5749 & 0.4671 \\
\hline 266.5983 & 2.9074 \\
\hline 298.3605 & 8.9865 \\
\hline 300.1119 & 0.8505 \\
\hline 307.3812 & 19.5028 \\
\hline 311.6979 & 5.5955 \\
\hline 332.1766 & 2.7658 \\
\hline 333.9221 & 1.8156 \\
\hline 337.8495 & 4.2775 \\
\hline 343.7232 & 24.2159 \\
\hline 355.9600 & 33.7924 \\
\hline 361.1956 & 0.5232 \\
\hline 366.0476 & 23.0407 \\
\hline 371.0470 & 7.7849 \\
\hline 382.7387 & 11.9755 \\
\hline 391.8215 & 0.3335 \\
\hline 394.8851 & 6.4782 \\
\hline 395.8668 & 0.4114 \\
\hline 402.6980 & 0.2457 \\
\hline 412.7555 & 0.1565 \\
\hline 423.8251 & 0.3730 \\
\hline 429.0988 & 0.2946 \\
\hline 436.4086 & 1.4883 \\
\hline 444.0903 & 0.5781 \\
\hline 444.1866 & 0.8474 \\
\hline 474.8339 & 1.7523 \\
\hline 479.6967 & 12.0850 \\
\hline 490.1043 & 10.9309 \\
\hline 504.9072 & 1.0901 \\
\hline 510.9328 & 21.4925 \\
\hline 519.1662 & 5.4865 \\
\hline 520.8137 & 12.5804 \\
\hline 535.8312 & 3.0169 \\
\hline 538.5525 & 5.0562 \\
\hline 551.7327 & 15.4361 \\
\hline 561.8681 & 58.7714 \\
\hline 569.6289 & 118.6869 \\
\hline 578.1689 & 137.6044 \\
\hline 634.1652 & 0.2751 \\
\hline 670.7672 & 12.7822 \\
\hline 672.8308 & 55.8563 \\
\hline 674.1501 & 64.9325 \\
\hline 676.8071 & 69.5860 \\
\hline 678.1419 & 68.4530 \\
\hline 683.6341 & 14.6724 \\
\hline 713.7199 & 50.9827 \\
\hline 771.6277 & 29.9067 \\
\hline 826.2422 & 8.3616 \\
\hline 863.3028 & 2.9070 \\
\hline
\end{tabular}




\begin{tabular}{|c|c|}
\hline 872.4470 & 98.4538 \\
\hline 942.7846 & 0.9723 \\
\hline 985.2682 & 10.1407 \\
\hline 994.4600 & 0.0111 \\
\hline 1015.7011 & 0.0801 \\
\hline 1017.4954 & 0.2539 \\
\hline 1049.1478 & 2.9578 \\
\hline 1110.2916 & 4.1631 \\
\hline 1185.9835 & 0.3072 \\
\hline 1203.9302 & 9.7198 \\
\hline 1212.3659 & 59.3298 \\
\hline 1226.5117 & 11.2222 \\
\hline 1258.6276 & 32.4575 \\
\hline 1344.6784 & 2.7791 \\
\hline 1363.5703 & 1.0086 \\
\hline 1391.8844 & 11.9373 \\
\hline 1485.7201 & 7.8069 \\
\hline 1487.5635 & 10.1681 \\
\hline 1526.5122 & 9.8589 \\
\hline 1545.8175 & 21.5219 \\
\hline 1546.0155 & 74.6533 \\
\hline 1550.7067 & 73.8231 \\
\hline 1552.0278 & 80.6151 \\
\hline 1558.5429 & 93.1202 \\
\hline 1560.3208 & 134.0415 \\
\hline 1564.4372 & 63.8085 \\
\hline 1569.9560 & 16.0409 \\
\hline 1573.9419 & 66.0594 \\
\hline 1581.6019 & 43.1929 \\
\hline 1583.2365 & 25.9918 \\
\hline 1586.9679 & 63.7384 \\
\hline 1624.3203 & 1.5045 \\
\hline 1643.0466 & 0.7464 \\
\hline 2255.0694 & 523.1580 \\
\hline 3036.2716 & 68.8314 \\
\hline 3096.2430 & 20.6899 \\
\hline 3159.0435 & 4.8622 \\
\hline 3167.7068 & 0.0946 \\
\hline 3175.5460 & 2.9703 \\
\hline 3186.1428 & 12.0990 \\
\hline 3195.2250 & 13.9812 \\
\hline
\end{tabular}


TS1

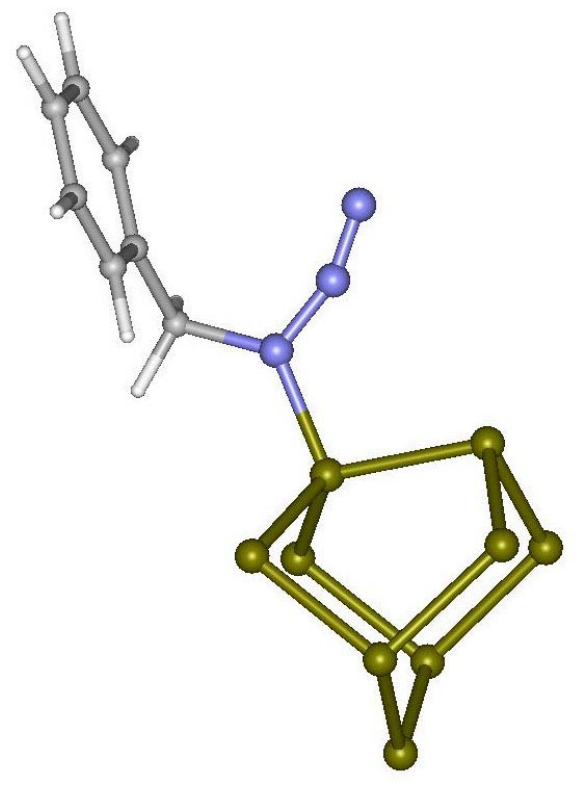

$$
\mathrm{E}(\mathrm{RB}+\mathrm{HF}-\mathrm{LYP})=-3048.15973570 \text { (Hartree/Particle })
$$

$\begin{array}{lrrr}\text { Si } & -4.005203 & 0.922929 & -1.203870 \\ \mathrm{Si} & -0.354075 & 2.024384 & -0.846780 \\ \mathrm{Si} & -2.182290 & 1.441981 & 2.180104 \\ \mathrm{Si} & -3.385025 & -1.364971 & -1.086132 \\ \mathrm{Si} & -1.106263 & -0.675544 & 1.938025 \\ \mathrm{Si} & -0.221872 & -0.244284 & -0.195954 \\ \mathrm{Si} & -2.969014 & -1.955842 & 1.170077 \\ \mathrm{Si} & -1.179709 & -1.558113 & -1.914112 \\ \mathrm{C} & 3.993126 & -0.199465 & -0.623902 \\ \mathrm{C} & 5.118911 & -1.006776 & -0.433906 \\ \mathrm{C} & 6.338715 & -0.442968 & -0.062216 \\ \mathrm{C} & 6.439306 & 0.932512 & 0.127587 \\ \mathrm{C} & 5.319212 & 1.745387 & -0.057230 \\ \mathrm{C} & 4.103603 & 1.184269 & -0.432167 \\ \mathrm{H} & 5.044864 & -2.078922 & -0.584549 \\ \mathrm{H} & 7.205356 & -1.078051 & 0.079449 \\ \mathrm{H} & 7.386526 & 1.372814 & 0.416939 \\ \mathrm{H} & 5.395852 & 2.816777 & 0.087288 \\ \mathrm{H} & 3.236272 & 1.819947 & -0.575728 \\ \mathrm{H} & -2.694247 & -3.424372 & 1.203103 \\ \mathrm{H} & -4.183338 & -1.733595 & 2.008932 \\ \mathrm{H} & 0.647597 & 2.874146 & -0.137114 \\ \mathrm{H} & -0.178131 & 2.241699 & -2.311264 \\ \mathrm{H} & -0.690587 & -2.967843 & -1.879055 \\ \mathrm{H} & -0.938598 & -1.023414 & -3.284677 \\ \mathrm{H} & -4.053264 & 1.359000 & -2.630358 \\ \mathrm{H} & -5.382425 & 1.077312 & -0.652950 \\ \mathrm{H} & -4.356022 & -2.191935 & -1.867425 \\ & & & \end{array}$




$\begin{array}{lrrr}\mathrm{H} & -2.986483 & 3.755462 & -0.104464 \\ \mathrm{H} & -3.470188 & 1.333298 & 2.926406 \\ \mathrm{H} & -1.338140 & 2.420680 & 2.930252 \\ \mathrm{~N} & 2.631892 & -1.694936 & 1.951295 \\ \mathrm{~N} & 1.971266 & -1.245001 & 1.154539 \\ \mathrm{~N} & 1.608362 & -0.746288 & 0.030386 \\ \mathrm{C} & 2.683935 & -0.810711 & -1.039657 \\ \mathrm{H} & 2.804725 & -1.856769 & -1.331793 \\ \mathrm{H} & 2.230609 & -0.271695 & -1.871937 \\ \mathrm{Si} & -2.535255 & 2.334143 & 0.012523\end{array}$




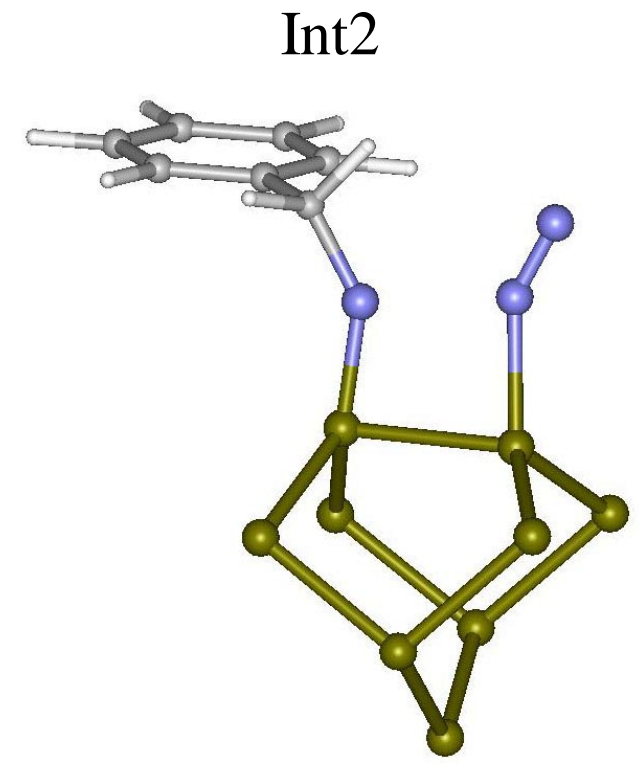

Zero-point correction=

0.240001 (Hartree/Particle) Sum of electronic and zero-point Energies= $-3047.962667$

$\begin{array}{lrrr}\mathrm{Si} & 3.638048 & 1.597899 & -0.605938 \\ \mathrm{Si} & 1.134462 & -0.624923 & -2.573956 \\ \mathrm{Si} & 3.003382 & -2.106128 & 0.245926 \\ \mathrm{Si} & 1.886643 & 2.373244 & 0.804280 \\ \mathrm{Si} & 1.140052 & -0.942980 & 1.097697 \\ \mathrm{Si} & -0.233148 & -0.183485 & -0.658941 \\ \mathrm{Si} & 1.634246 & 0.814646 & 2.585868 \\ \mathrm{Si} & -0.202594 & 2.184221 & -0.316602 \\ \mathrm{C} & -3.811034 & -0.667970 & -0.236025 \\ \mathrm{C} & -4.547267 & -0.348354 & -1.379013 \\ \mathrm{C} & -5.536111 & 0.636801 & -1.342451 \\ \mathrm{C} & -5.801356 & 1.314679 & -0.155508 \\ \mathrm{C} & -5.072595 & 1.001788 & 0.993805 \\ \mathrm{C} & -4.086189 & 0.020928 & 0.950817 \\ \mathrm{H} & -4.347741 & -0.876535 & -2.306635 \\ \mathrm{H} & -6.097481 & 0.871827 & -2.240327 \\ \mathrm{H} & -6.570064 & 2.078708 & -0.122803 \\ \mathrm{H} & -5.277718 & 1.521636 & 1.923540 \\ \mathrm{H} & -3.518805 & -0.216401 & 1.844934 \\ \mathrm{H} & 0.454222 & 1.134863 & 3.432429 \\ \mathrm{H} & 2.824419 & 0.606030 & 3.452614 \\ \mathrm{H} & 0.910198 & -1.985950 & -3.138390 \\ \mathrm{H} & 1.047947 & 0.363837 & -3.686126 \\ \mathrm{H} & -1.281865 & 2.654877 & 0.594266 \\ \mathrm{H} & -0.220832 & 3.021296 & -1.549444 \\ \mathrm{H} & 3.803817 & 2.567896 & -1.725363 \\ \mathrm{H} & 4.912649 & 1.580642 & 0.167891 \\ \mathrm{H} & 2.257859 & 3.730769 & 1.301808 \\ \mathrm{H} & 4.443290 & -0.898271 & -2.403624 \\ \mathrm{H} & 4.154323 & -2.241048 & 1.177637\end{array}$




$\begin{array}{lrrr}\mathrm{H} & 2.602863 & -3.450333 & -0.248422 \\ \mathrm{~N} & -0.881873 & -2.820532 & 2.251180 \\ \mathrm{~N} & -0.400018 & -2.057611 & 1.579949 \\ \mathrm{~N} & -1.398799 & -1.240245 & -0.059623 \\ \mathrm{C} & -2.720357 & -1.729558 & -0.280755 \\ \mathrm{H} & -2.807911 & -2.287543 & -1.226127 \\ \mathrm{H} & -2.921631 & -2.471197 & 0.517814 \\ \mathrm{Si} & 3.273676 & -0.558655 & -1.540579\end{array}$

Frequency

\begin{tabular}{|c|c|}
\hline & \\
\hline 10.7927 & 0.4432 \\
\hline 17.8648 & 0.0136 \\
\hline 24.6789 & 0.0089 \\
\hline 47.9095 & 0.4727 \\
\hline 70.0132 & 1.2234 \\
\hline 73.2319 & 0.3524 \\
\hline 81.8187 & 5.3509 \\
\hline 94.5160 & 0.0751 \\
\hline 100.9081 & 0.3667 \\
\hline 104.6519 & 1.3938 \\
\hline 118.7587 & 3.3722 \\
\hline 126.7323 & 4.6383 \\
\hline 145.4734 & 3.6046 \\
\hline 153.1674 & 1.4150 \\
\hline 172.1217 & 10.0724 \\
\hline 176.2911 & 1.4000 \\
\hline 193.6336 & 7.7315 \\
\hline 236.8033 & 3.4841 \\
\hline 264.3673 & 4.6973 \\
\hline 279.9665 & 18.1724 \\
\hline 316.3871 & 6.2774 \\
\hline 320.0997 & 0.8252 \\
\hline 323.7856 & 2.6628 \\
\hline 344.6623 & 17.1904 \\
\hline 357.4545 & 0.3187 \\
\hline 360.3550 & 2.0868 \\
\hline 377.6813 & 2.9542 \\
\hline 385.6494 & 4.0276 \\
\hline 399.5593 & 12.4093 \\
\hline 407.7268 & 2.2467 \\
\hline 408.5899 & 2.1656 \\
\hline 412.3165 & 0.2675 \\
\hline 415.3897 & 0.4649 \\
\hline 417.8422 & 2.8857 \\
\hline 443.7791 & 6.3663 \\
\hline 453.9048 & 10.5197 \\
\hline 456.0722 & 0.4239 \\
\hline 471.8343 & 1.8565 \\
\hline 480.9872 & 1.8590 \\
\hline
\end{tabular}




\begin{tabular}{|c|c|}
\hline 485.9991 & 4.7320 \\
\hline 505.0593 & 32.3404 \\
\hline 515.8552 & 52.9282 \\
\hline 561.7057 & 0.5268 \\
\hline 565.2962 & 6.4548 \\
\hline 593.0269 & 7.6693 \\
\hline 595.1648 & 1.5269 \\
\hline 601.6377 & 14.6560 \\
\hline 613.6738 & 3.2996 \\
\hline 624.6103 & 1.1156 \\
\hline 634.3446 & 0.6344 \\
\hline 635.8557 & 1.7189 \\
\hline 644.9875 & 3.6751 \\
\hline 656.8508 & 3.0557 \\
\hline 670.9483 & 82.6920 \\
\hline 675.7629 & 0.7554 \\
\hline 690.2317 & 19.0472 \\
\hline 707.1443 & 105.2507 \\
\hline 711.2166 & 14.2858 \\
\hline 730.8734 & 219.1995 \\
\hline 735.9916 & 255.7700 \\
\hline 777.8426 & 21.2624 \\
\hline 819.2400 & 6.2021 \\
\hline 852.8443 & 0.5250 \\
\hline 920.6756 & 3.7502 \\
\hline 924.6437 & 77.8274 \\
\hline 924.9593 & 44.3704 \\
\hline 927.8679 & 173.5876 \\
\hline 928.5815 & 262.7575 \\
\hline 939.7937 & 29.3398 \\
\hline 969.9978 & 1.7049 \\
\hline 980.8582 & 0.1605 \\
\hline 998.7832 & 0.3614 \\
\hline 1016.8785 & 0.3284 \\
\hline 1047.4876 & 7.1763 \\
\hline 1101.3386 & 6.2008 \\
\hline 1179.4121 & 0.0275 \\
\hline 1197.6159 & 5.3479 \\
\hline 1205.9106 & 14.8138 \\
\hline 1238.7739 & 12.1710 \\
\hline 1251.3443 & 245.3974 \\
\hline 1335.4983 & 9.0730 \\
\hline 1348.3395 & 14.3443 \\
\hline 1365.2659 & 48.2775 \\
\hline 1475.0928 & 4.7861 \\
\hline 1481.6062 & 9.8870 \\
\hline 1522.3427 & 10.0281 \\
\hline 1622.4597 & 1.0474 \\
\hline 1639.3659 & 9.9877 \\
\hline
\end{tabular}




\begin{tabular}{|l|l|}
\hline 2134.9621 & 285.7342 \\
\hline 2169.1847 & 69.4394 \\
\hline 2174.4280 & 56.5804 \\
\hline 2182.5786 & 135.4763 \\
\hline 2183.7447 & 124.5411 \\
\hline 2187.9222 & 134.9337 \\
\hline 2195.5060 & 40.7347 \\
\hline 2197.9552 & 107.1117 \\
\hline 2202.1599 & 81.2521 \\
\hline 2204.2952 & 123.8102 \\
\hline 2207.2991 & 93.7637 \\
\hline 2217.8089 & 34.4990 \\
\hline 2219.6712 & 115.3269 \\
\hline 2886.3337 & 65.8218 \\
\hline 2952.9823 & 78.9003 \\
\hline 3150.2152 & 6.7737 \\
\hline 3156.1538 & 2.0704 \\
\hline 3166.2615 & 7.1363 \\
\hline 3185.9692 & 32.1153 \\
\hline & 22.0931 \\
\hline
\end{tabular}

Deuterium-substituted:

Frequency

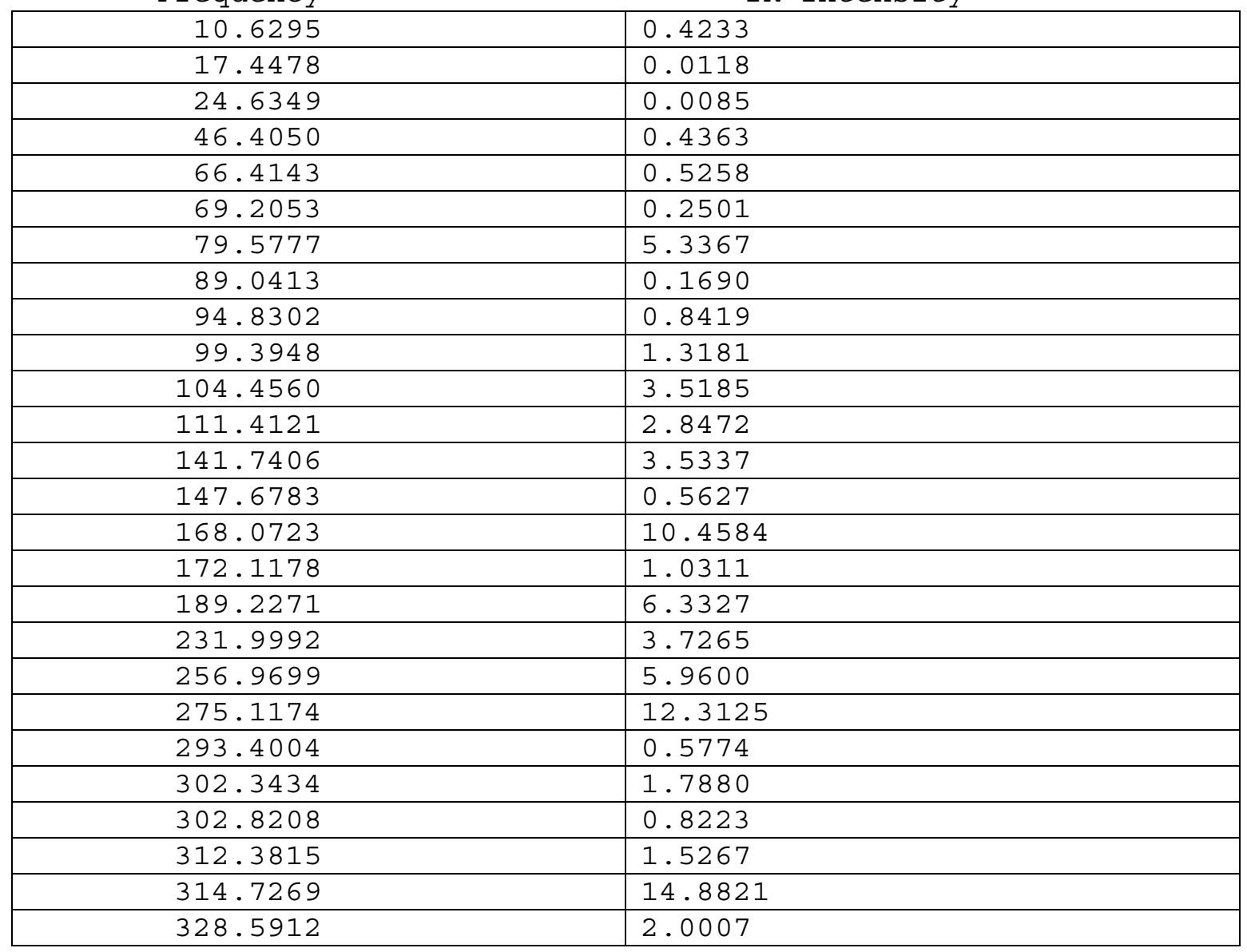




\begin{tabular}{|c|c|}
\hline 332.9070 & 4.4290 \\
\hline 335.6723 & 2.6878 \\
\hline 339.6579 & 1.8154 \\
\hline 365.5237 & 1.3098 \\
\hline 366.0968 & 2.4903 \\
\hline 368.4048 & 2.5100 \\
\hline 382.8433 & 0.8931 \\
\hline 386.0546 & 1.2313 \\
\hline 388.6639 & 0.0324 \\
\hline 394.8437 & 0.2831 \\
\hline 398.4774 & 1.0342 \\
\hline 400.3388 & 24.5921 \\
\hline 412.3024 & 0.2568 \\
\hline 417.0342 & 5.0126 \\
\hline 421.1691 & 0.9615 \\
\hline 432.8433 & 0.2703 \\
\hline 443.0970 & 5.3158 \\
\hline 445.7388 & 4.4196 \\
\hline 479.3268 & 25.6089 \\
\hline 490.4114 & 13.6464 \\
\hline 491.3247 & 31.2660 \\
\hline 504.6401 & 4.0034 \\
\hline 508.9399 & 10.7150 \\
\hline 513.5550 & 42.2387 \\
\hline 520.5374 & 0.3874 \\
\hline 524.1486 & 140.2839 \\
\hline 528.7491 & 0.0417 \\
\hline 546.2875 & 5.3899 \\
\hline 551.4704 & 90.4077 \\
\hline 567.7597 & 115.0813 \\
\hline 603.7211 & 28.2889 \\
\hline 634.9746 & 0.2163 \\
\hline 668.8721 & 43.1680 \\
\hline 670.3044 & 15.7587 \\
\hline 671.6921 & 122.5000 \\
\hline 673.3162 & 70.3366 \\
\hline 679.0858 & 22.5670 \\
\hline 707.0651 & 32.2095 \\
\hline 713.6987 & 16.4705 \\
\hline 776.7850 & 37.7966 \\
\hline 819.2036 & 5.9804 \\
\hline 852.8456 & 0.4269 \\
\hline 920.7403 & 6.1909 \\
\hline 969.9660 & 1.4420 \\
\hline 980.8556 & 0.1885 \\
\hline 998.7813 & 0.3737 \\
\hline 1016.8765 & 0.3764 \\
\hline 1047.4806 & 7.1548 \\
\hline 1101.3335 & 6.2311 \\
\hline
\end{tabular}




\begin{tabular}{|c|c|}
\hline 1179.4119 & 0.0265 \\
\hline 1197.6080 & 5.6025 \\
\hline 1205.8809 & 15.3705 \\
\hline 1238.7421 & 13.1782 \\
\hline 1251.0377 & 250.4245 \\
\hline 1335.4951 & 9.0467 \\
\hline 1348.3337 & 14.4042 \\
\hline 1365.2458 & 48.2986 \\
\hline 1475.0896 & 4.6596 \\
\hline 1481.6032 & 9.7772 \\
\hline 1522.3405 & 9.9628 \\
\hline 1557.9173 & 3.1162 \\
\hline 1560.3720 & 15.1293 \\
\hline 1563.4412 & 71.5000 \\
\hline 1564.2859 & 96.1630 \\
\hline 1568.6083 & 107.0701 \\
\hline 1575.2994 & 71.3937 \\
\hline 1577.4175 & 45.0257 \\
\hline 1586.1396 & 22.5692 \\
\hline 1588.5309 & 46.9687 \\
\hline 1594.0705 & 40.0988 \\
\hline 1603.5654 & 14.2630 \\
\hline 1605.0392 & 54.5709 \\
\hline 1622.4622 & 1.0391 \\
\hline 1639.3706 & 10.0632 \\
\hline 2135.0188 & 283.2660 \\
\hline 2886.3322 & 65.9836 \\
\hline 2952.9794 & 78.8683 \\
\hline 3150.2152 & 6.7633 \\
\hline 3156.1537 & 2.0724 \\
\hline 3166.2614 & 7.1427 \\
\hline 3173.9475 & 32.0775 \\
\hline 3185.9691 & 22.0963 \\
\hline
\end{tabular}




\section{TS2}

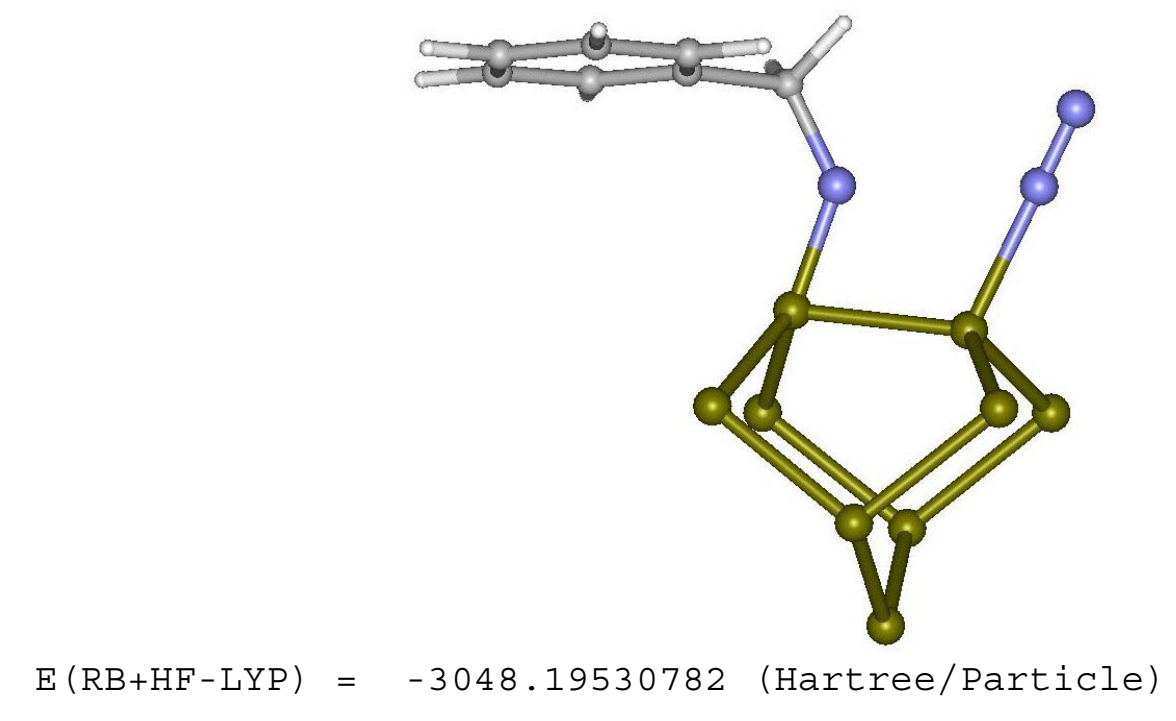

$\begin{array}{lrrr}\mathrm{Si} & 3.476603 & -1.771471 & .223456 \\ \mathrm{Si} & -.363746 & -2.123502 & .737695 \\ \mathrm{Si} & 1.519424 & .472079 & 2.645106 \\ \mathrm{Si} & 3.149667 & -.331031 & -1.641952 \\ \mathrm{Si} & 1.054749 & 1.284018 & .480345 \\ \mathrm{Si} & -.294677 & -.184892 & -.669792 \\ \mathrm{Si} & 2.937398 & 1.885829 & -.805352 \\ \mathrm{Si} & 1.014390 & -.760311 & -2.599645 \\ \mathrm{C} & -3.642237 & .587252 & -.510817 \\ \mathrm{C} & -4.129846 & .564731 & .801430 \\ \mathrm{C} & -5.070157 & -.381473 & 1.199424 \\ \mathrm{C} & -5.538750 & -1.330647 & .289145 \\ \mathrm{C} & -5.062782 & -1.320118 & -1.019838 \\ \mathrm{C} & -4.123658 & -.365883 & -1.414507 \\ \mathrm{H} & -3.765565 & 1.297341 & 1.514896 \\ \mathrm{H} & -5.441421 & -.379304 & 2.218728 \\ \mathrm{H} & -6.271139 & -2.068112 & .598113 \\ \mathrm{H} & -5.425634 & -2.049435 & -1.736157 \\ \mathrm{H} & -3.767637 & -.354324 & -2.440632 \\ \mathrm{H} & 2.567686 & 2.852508 & -1.874528 \\ \mathrm{H} & 4.107472 & 2.415798 & -.053175 \\ \mathrm{H} & -1.451466 & -2.089238 & 1.753714 \\ \mathrm{H} & -.375790 & -3.459688 & .075298 \\ \mathrm{H} & .803980 & .168296 & -3.747238 \\ \mathrm{H} & .941401 & -2.158366 & -3.115028 \\ \mathrm{H} & 3.628227 & -3.164070 & -.286780 \\ \mathrm{H} & 4.754206 & -1.401552 & .898972 \\ \mathrm{H} & 4.327888 & -.470696 & -2.548647 \\ \mathrm{H} & 2.083696 & -2.709395 & 2.916918 \\ \mathrm{H} & 2.720629 & 1.034640 & 3.320529 \\ \mathrm{H} & .339843 & .631182 & 3.538304\end{array}$




$\begin{array}{lrlr}\mathrm{N} & -.559602 & 3.972733 & .908398 \\ \mathrm{~N} & .014710 & 3.041499 & .771518 \\ \mathrm{~N} & -1.238000 & 1.209144 & -.510441 \\ \mathrm{C} & -2.573382 & 1.582362 & -.927441 \\ \mathrm{H} & -2.809945 & 2.562182 & -.480257 \\ \mathrm{H} & -2.635426 & 1.728890 & -2.018027 \\ \mathrm{Si} & 1.720057 & -1.754120 & 1.828305\end{array}$




\section{Product}

\begin{tabular}{|c|c|c|c|}
\hline & $\begin{array}{l}\text { int correct } \\
\text { electronic }\end{array}$ & $\begin{array}{l}\text { on }= \\
\text { and zero-poi }\end{array}$ & $\begin{array}{l}0.233363 \\
\text { Energies }\end{array}$ \\
\hline Si & 3.616412 & 0.406808 & -1.046156 \\
\hline Si & 0.709041 & -2.060892 & -1.610916 \\
\hline $\mathrm{Si}$ & 2.168016 & -1.903011 & 1.668924 \\
\hline Si & 2.200006 & 2.255619 & -0.519546 \\
\hline Si & 0.660071 & -0.099842 & 1.570292 \\
\hline Si & -0.293100 & -0.203325 & -0.571855 \\
\hline Si & 1.559360 & 2.063073 & 1.751958 \\
\hline Si & 0.101024 & 1.891903 & -1.554295 \\
\hline C & -3.457205 & -0.375592 & 0.746011 \\
\hline C & -4.093820 & -1.343666 & -0.035373 \\
\hline $\mathrm{C}$ & -5.171227 & -1.004839 & -0.854760 \\
\hline C & -5.623786 & 0.311242 & -0.901595 \\
\hline $\mathrm{C}$ & -4.995074 & 1.286716 & -0.126096 \\
\hline C & -3.920138 & 0.944539 & 0.689112 \\
\hline $\mathrm{H}$ & -3.748377 & -2.371996 & 0.002590 \\
\hline $\mathrm{H}$ & -5.656217 & -1.768679 & -1.452396 \\
\hline $\mathrm{H}$ & -6.462689 & 0.576772 & -1.535057 \\
\hline $\mathrm{H}$ & -5.346078 & 2.312326 & -0.155233 \\
\hline $\mathrm{H}$ & -3.433841 & 1.705865 & 1.290381 \\
\hline $\mathrm{H}$ & 0.550889 & 3.085859 & 2.144956 \\
\hline $\mathrm{H}$ & 2.702712 & 2.120598 & 2.704671 \\
\hline $\mathrm{H}$ & 0.072421 & -3.358881 & -1.251954 \\
\hline $\mathrm{H}$ & 0.725543 & -1.927678 & -3.093962 \\
\hline $\mathrm{H}$ & -0.902223 & 2.926158 & -1.182290 \\
\hline $\mathrm{H}$ & 0.181548 & 1.803167 & -3.038736 \\
\hline $\mathrm{H}$ & 3.947659 & 0.487693 & -2.499487 \\
\hline $\mathrm{H}$ & 4.900014 & 0.590187 & -0.306488 \\
\hline
\end{tabular}




$\begin{array}{lccc}\mathrm{H} & 2.916090 & 3.515583 & -0.899313 \\ \mathrm{H} & 3.897560 & -2.770234 & -1.025517 \\ \mathrm{H} & 3.272308 & -1.648879 & 2.635333 \\ \mathrm{H} & 1.521092 & -3.200214 & 2.011365 \\ \mathrm{~N} & -1.002589 & -0.302312 & 1.031630 \\ \mathrm{C} & -2.277109 & -0.738706 & 1.617985 \\ \mathrm{H} & -2.271099 & -1.822812 & 1.785932 \\ \mathrm{H} & -2.380731 & -0.260953 & 2.598311 \\ \mathrm{Si} & 2.833576 & -1.804324 & -0.600279\end{array}$

\section{Frequency}

\begin{tabular}{|c|c|}
\hline 9.8997 & 0.0201 \\
\hline 19.6709 & 0.1093 \\
\hline 30.7241 & 0.1394 \\
\hline 67.0082 & 0.4428 \\
\hline 76.3838 & 0.1727 \\
\hline 86.5565 & 0.0592 \\
\hline 92.7249 & 0.5405 \\
\hline 109.0456 & 1.3590 \\
\hline 111.0946 & 1.3027 \\
\hline 122.9849 & 3.5911 \\
\hline 127.9460 & 0.8498 \\
\hline 150.6988 & 0.0238 \\
\hline 191.8322 & 0.3376 \\
\hline 195.1928 & 4.2390 \\
\hline 222.0264 & 2.4892 \\
\hline 227.9203 & 12.2514 \\
\hline 238.4052 & 2.3494 \\
\hline 315.6673 & 1.3817 \\
\hline 317.1652 & 0.1935 \\
\hline 325.7898 & 0.3211 \\
\hline 340.4605 & 0.2735 \\
\hline 363.1823 & 1.2511 \\
\hline 367.4255 & 1.8105 \\
\hline 376.5356 & 0.0101 \\
\hline 385.0009 & 1.4499 \\
\hline 401.3676 & 0.0059 \\
\hline 408.6090 & 0.2500 \\
\hline 412.7573 & 0.1165 \\
\hline 414.9364 & 0.1047 \\
\hline 415.8320 & 0.0029 \\
\hline 456.8021 & 0.5111 \\
\hline 457.5658 & 1.5337 \\
\hline 467.4770 & 2.0917 \\
\hline 485.3432 & 1.0074 \\
\hline 489.7996 & 9.7113 \\
\hline 509.2402 & 9.6413 \\
\hline 581.9734 & 0.1136 \\
\hline 583.8404 & 2.0072 \\
\hline 596.2997 & 5.2299 \\
\hline
\end{tabular}




\begin{tabular}{|c|c|}
\hline 610.8470 & 2.7648 \\
\hline 617.2074 & 0.0056 \\
\hline 623.0478 & 0.4856 \\
\hline 623.9293 & 0.4396 \\
\hline 635.4299 & 0.0250 \\
\hline 643.6064 & 0.1109 \\
\hline 652.6446 & 16.0567 \\
\hline 656.3989 & 25.4873 \\
\hline 676.5340 & 4.1180 \\
\hline 683.3879 & 1.5880 \\
\hline 694.3855 & 0.2146 \\
\hline 698.9527 & 29.1318 \\
\hline 709.3666 & 38.7173 \\
\hline 727.5451 & 203.1067 \\
\hline 734.6380 & 77.1547 \\
\hline 747.7458 & 284.9816 \\
\hline 786.6667 & 17.9149 \\
\hline 842.7505 & 4.3653 \\
\hline 856.8359 & 0.6146 \\
\hline 918.7677 & 1.7150 \\
\hline 921.0078 & 280.5607 \\
\hline 921.9313 & 133.3925 \\
\hline 923.0367 & 123.8810 \\
\hline 932.3036 & 21.7838 \\
\hline 934.4195 & 33.3281 \\
\hline 981.5802 & 0.9473 \\
\hline 985.7401 & 0.1616 \\
\hline 1005.1549 & 0.4929 \\
\hline 1017.7653 & 0.7514 \\
\hline 1048.5275 & 7.1440 \\
\hline 1104.7656 & 10.3628 \\
\hline 1152.1250 & 254.2650 \\
\hline 1181.7681 & 0.0364 \\
\hline 1201.1612 & 0.1020 \\
\hline 1223.6212 & 4.6500 \\
\hline 1232.5069 & 12.7470 \\
\hline 1339.5262 & 1.8281 \\
\hline 1354.2016 & 5.3766 \\
\hline 1372.1782 & 22.9965 \\
\hline 1482.2295 & 6.0997 \\
\hline 1505.4057 & 3.6484 \\
\hline 1525.6889 & 4.1728 \\
\hline 1623.6080 & 1.0173 \\
\hline 1642.9761 & 1.0559 \\
\hline 2142.8235 & 161.6043 \\
\hline 2144.2295 & 189.6932 \\
\hline 2175.9594 & 173.4770 \\
\hline 2184.1452 & 11.9397 \\
\hline 2188.2040 & 39.6833 \\
\hline
\end{tabular}




\begin{tabular}{|l|l|}
\hline 2189.5989 & 205.3028 \\
\hline 2191.5408 & 245.1072 \\
\hline 2195.0107 & 10.5860 \\
\hline 2203.6235 & 40.4745 \\
\hline 2205.8778 & 111.8441 \\
\hline 2206.8105 & 94.4953 \\
\hline 2211.3112 & 103.2172 \\
\hline 2997.5335 & 70.4712 \\
\hline 3036.7928 & 13.1956 \\
\hline 3156.4290 & 5.3749 \\
\hline 3160.0699 & 0.9857 \\
\hline 3169.7489 & 8.0216 \\
\hline 3178.5776 & 23.6631 \\
\hline & 16.7686 \\
\hline
\end{tabular}

Deuterium-substituted:

\section{Frequency}

\begin{tabular}{|c|c|}
\hline 9.5439 & 0.0194 \\
\hline 19.5998 & 0.1048 \\
\hline 30.0894 & 0.1334 \\
\hline 63.2406 & 0.3188 \\
\hline 71.7094 & 0.3035 \\
\hline 77.9645 & 0.0633 \\
\hline 83.4416 & 0.5178 \\
\hline 96.1909 & 1.5531 \\
\hline 105.7199 & 2.9199 \\
\hline 107.5514 & 0.1383 \\
\hline 125.7048 & 0.6857 \\
\hline 145.5897 & 0.0214 \\
\hline 188.3028 & 0.1230 \\
\hline 190.8430 & 3.6941 \\
\hline 215.6208 & 10.4436 \\
\hline 219.2013 & 1.7348 \\
\hline 232.6921 & 1.0857 \\
\hline 288.8055 & 0.0099 \\
\hline 296.1765 & 0.0110 \\
\hline 299.5647 & 3.2603 \\
\hline 308.5628 & 0.5791 \\
\hline 317.5132 & 0.1144 \\
\hline 330.7883 & 1.7421 \\
\hline 335.0150 & 1.2143 \\
\hline 342.5590 & 4.9188 \\
\hline 345.3732 & 1.0692 \\
\hline 360.5948 & 0.1184 \\
\hline 371.2126 & 0.3519 \\
\hline 373.8626 & 0.0009 \\
\hline 386.0826 & 0.4385 \\
\hline
\end{tabular}




\begin{tabular}{|c|c|}
\hline 391.9510 & 1.9249 \\
\hline 400.5599 & 0.3852 \\
\hline 402.0002 & 0.0224 \\
\hline 412.7569 & 0.1258 \\
\hline 423.8137 & 0.0068 \\
\hline 431.5340 & 0.0255 \\
\hline 446.8131 & 0.0175 \\
\hline 447.7835 & 1.9625 \\
\hline 451.5018 & 0.9926 \\
\hline 454.7631 & 6.3338 \\
\hline 489.7120 & 21.4904 \\
\hline 492.2294 & 23.6057 \\
\hline 502.8038 & 45.7031 \\
\hline 518.1579 & 4.1376 \\
\hline 531.2608 & 0.0711 \\
\hline 540.0926 & 1.0569 \\
\hline 541.3836 & 0.6652 \\
\hline 542.5074 & 42.2016 \\
\hline 550.3759 & 96.8362 \\
\hline 575.2242 & 125.4970 \\
\hline 590.6009 & 0.7539 \\
\hline 635.1791 & 0.1112 \\
\hline 663.3963 & 37.8629 \\
\hline 666.8679 & 23.5305 \\
\hline 667.2784 & 102.2316 \\
\hline 669.6372 & 75.5675 \\
\hline 671.7853 & 42.8740 \\
\hline 678.5194 & 5.6400 \\
\hline 708.2925 & 29.5573 \\
\hline 729.1880 & 42.1130 \\
\hline 785.8314 & 23.0436 \\
\hline 842.4750 & 4.8035 \\
\hline 856.8510 & 0.4746 \\
\hline 932.3670 & 9.0229 \\
\hline 981.5102 & 0.9279 \\
\hline 985.7360 & 0.2014 \\
\hline 1005.1533 & 0.5214 \\
\hline 1017.7607 & 0.7894 \\
\hline 1048.5237 & 7.1959 \\
\hline 1104.7593 & 10.5289 \\
\hline 1151.9443 & 257.6389 \\
\hline 1181.7678 & 0.0390 \\
\hline 1201.1585 & 0.1116 \\
\hline 1223.6122 & 4.7207 \\
\hline 1232.4934 & 12.7978 \\
\hline 1339.5233 & 1.8514 \\
\hline 1354.1941 & 5.4848 \\
\hline 1372.1392 & 23.3514 \\
\hline 1482.2271 & 6.0331 \\
\hline
\end{tabular}




\begin{tabular}{|c|c|}
\hline 1505.3915 & 3.6829 \\
\hline 1525.6866 & 4.3113 \\
\hline 1540.5380 & 57.4567 \\
\hline 1541.9619 & 86.6142 \\
\hline 1557.4963 & 110.4512 \\
\hline 1565.1789 & 21.4463 \\
\hline 1566.5507 & 139.5911 \\
\hline 1567.5589 & 112.2324 \\
\hline 1571.2041 & 7.5585 \\
\hline 1578.1027 & 43.7852 \\
\hline 1592.7378 & 12.3031 \\
\hline 1594.4873 & 44.4822 \\
\hline 1595.3738 & 46.2229 \\
\hline 1598.3088 & 50.9462 \\
\hline 1623.6118 & 0.9056 \\
\hline 1642.9782 & 1.0194 \\
\hline 2997.5302 & 70.4873 \\
\hline 3036.7918 & 13.1229 \\
\hline 3156.4289 & 5.3717 \\
\hline 3160.0698 & 0.9811 \\
\hline 3169.7488 & 8.0311 \\
\hline 3178.5775 & 23.6120 \\
\hline 3189.1532 & 16.7775 \\
\hline
\end{tabular}

